\title{
Meeting women's health care needs after abortion
}

Dale Huntington

Follow this and additional works at: https://knowledgecommons.popcouncil.org/departments_sbsr-rh

Part of the Family, Life Course, and Society Commons, International Public Health Commons, and the Women's Health Commons

How does access to this work benefit you? Let us know!

\section{Recommended Citation}

Huntington, Dale. 2000. "Meeting women's health care needs after abortion," FRONTIERS Program Brief. Washington, DC: Population Council. 


\section{Meeting Women's Health Care Needs After Abortion}

\section{The Postabortion Care Challenge}

$\sqrt{1}$ omen who seek emergency treatment for abortion complications - bleeding, infection and injuries to the reproductive tract system - should be a priority group for reproductive health care programs. These women often receive poor-quality services that do not address their multiple health needs. They may be discharged without counseling on postoperative recuperation, family planning, or other reproductive health issues.

Women who have had an induced abortion due to an unwanted pregnancy are likely to have a repeat abortion unless they receive appropriate family planning counseling and services. Preventing repeat unsafe abortions is important for reproductive health programs because it saves women's lives, protects women's health, and reduces the need for costly emergency services for abortion complications.

At the 1994 International Conference on Population and Development, the world's governments called for improvements in postabortion medical services. As part of the resulting international postabortion

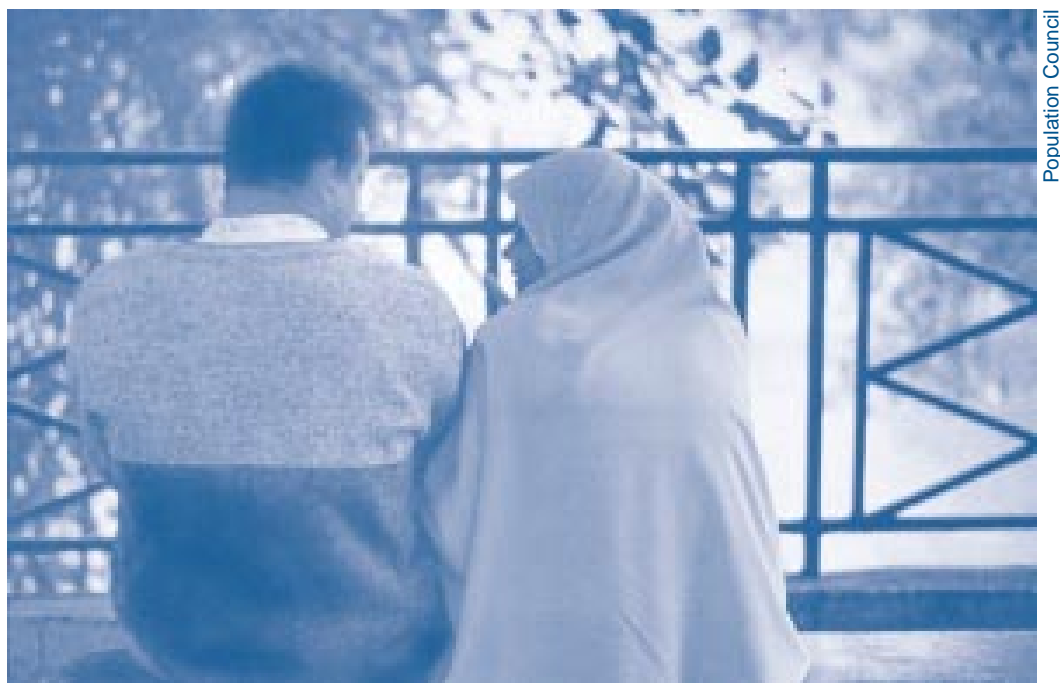

Postabortion care is a service delivery strategy built around three elements:

\section{Emergency treatment of medical complications of spontaneous abortion (miscarriage) or induced abortion}

\section{Postabortion family planning counseling, serv- ices, and referral}

\section{Linkages to other reproductive health services.}

care initiative, the Population Council's Operations Research and Technical Assistance (OR/TA) projects worked collaboratively with Ipas, the Johns Hopkins Program for International Education in Gynecology and Obstetrics
(JHPIEGO), and AVSC International to conduct research on interventions to improve postabortion care. This Program Brief summarizes the major findings of this research and relevant studies by other international organizations. 


\section{Why is postabortion care (PAC) important?}

PAC protects women's health. One in eight pregnancy-related deaths worldwide is caused by unsafe abortion. Many of these deaths - 13 percent of all maternal deaths - could be prevented by implementing appropriate postabortion care and helping couples obtain family planning information and services.

\section{PAC reduces women's suffering.}

Most women suffer physical pain and emotional trauma from unsafe abortion and miscarriage. Their suffering could be reduced by provision of compassionate, high-quality medical care.

\section{PAC reduces health care costs.}

Complications from unsafe abortions represent a significant cost to health care systems in terms of personnel time, supplies, anesthesia, blood transfusions, antibiotics, hospital beds, and operating rooms.

\begin{tabular}{|c|c|}
\hline \multicolumn{2}{|l|}{ Contents } \\
\hline Quality of Care & 4 \\
\hline Family Planning & 7 \\
\hline Access & 8 \\
\hline Planning Services & 9 \\
\hline Male Partners & 10 \\
\hline Sustainability & 10 \\
\hline Supportive Policies & 11 \\
\hline Research Priorities & 11 \\
\hline $\begin{array}{l}\text { Resources for } \\
\quad \text { Program Managers }\end{array}$ & 13 \\
\hline
\end{tabular}

\section{The World's Governments Endorse Postabortion Care}

All governments and relevant intergovernmental and nongovernmental organizations are urged to strengthen their commitment to women's health, to deal with the health impact of unsafe abortion as a major public health concern and to reduce the recourse to abortion through expanded and improved family planning services. Prevention of unwanted pregnancies must always be given the highest priority and every attempt should be made to eliminate the need for abortion. Women who have unwanted pregnancies should have ready access to reliable information and compassionate counseling... In all cases women should have access to quality services for the management of complications arising from abortion.

-Programme of Action, United Nations International Conference on Population and Development, Cairo, 1994, paragraph 8.25 (UN 1994).

PAC improves the quality
of health care provided to

women. Many women requiring emergency treatment for abortion complications receive poor care due to misdiagnosis, hospital overloads, punitive or negative attitudes, lack of necessary equipment and supplies, and the absence of medical protocols for postabortion care. Correcting these problems benefits all women with pregnancy-related emergencies.

PAC offers opportunities to meet the multiple health needs of underserved women. For some women, the first contact with the formal health care system occurs during emergency treatment of abortion complications. Thus, PAC offers an opportunity to inform women about available family planning (FP) choices, connect patients with other types of reproductive health (RH) care, and improve women's overall health.

\section{PAC can prevent repeat}

abortions. Studies in several countries found that between 11 and 48 percent of the women seeking emergency treatment for abortion complications have already had a previous abortion (Salter, Johnston, and Hengen 1997). Most women who have had one or more induced abortions are likely to be motivated to prevent future pregnancies. Providing family planning counseling and services can help them to prevent future unwanted pregnancies and reduce their need for abortions and subsequent emergency postabortion care. 


\section{Improving Postabortion Care}

Recent studies on interventions to improve postabortion services support several key observations:

PAC is a global issue. Poorquality postabortion care service is a widespread, multi-dimensional problem that affects women in many countries regardless of the legal status of abortion.

Training and supervision have a major impact. Technical and interpersonal skills training for clinicians and regular supervision can vastly improve the quality of postabortion care services.
PAC patients are interested in family planning. If postabortion care patients are given appropriate counseling and access to family planning services, many of them will start using contraception.

\section{Providers can reduce} treatment costs. Various measures, such as streamlining patient management, providing outpatient care for appropriate cases, and using manual vacuum aspiration (MVA) under local anesthesia, can reduce costs while improving the quality of care.

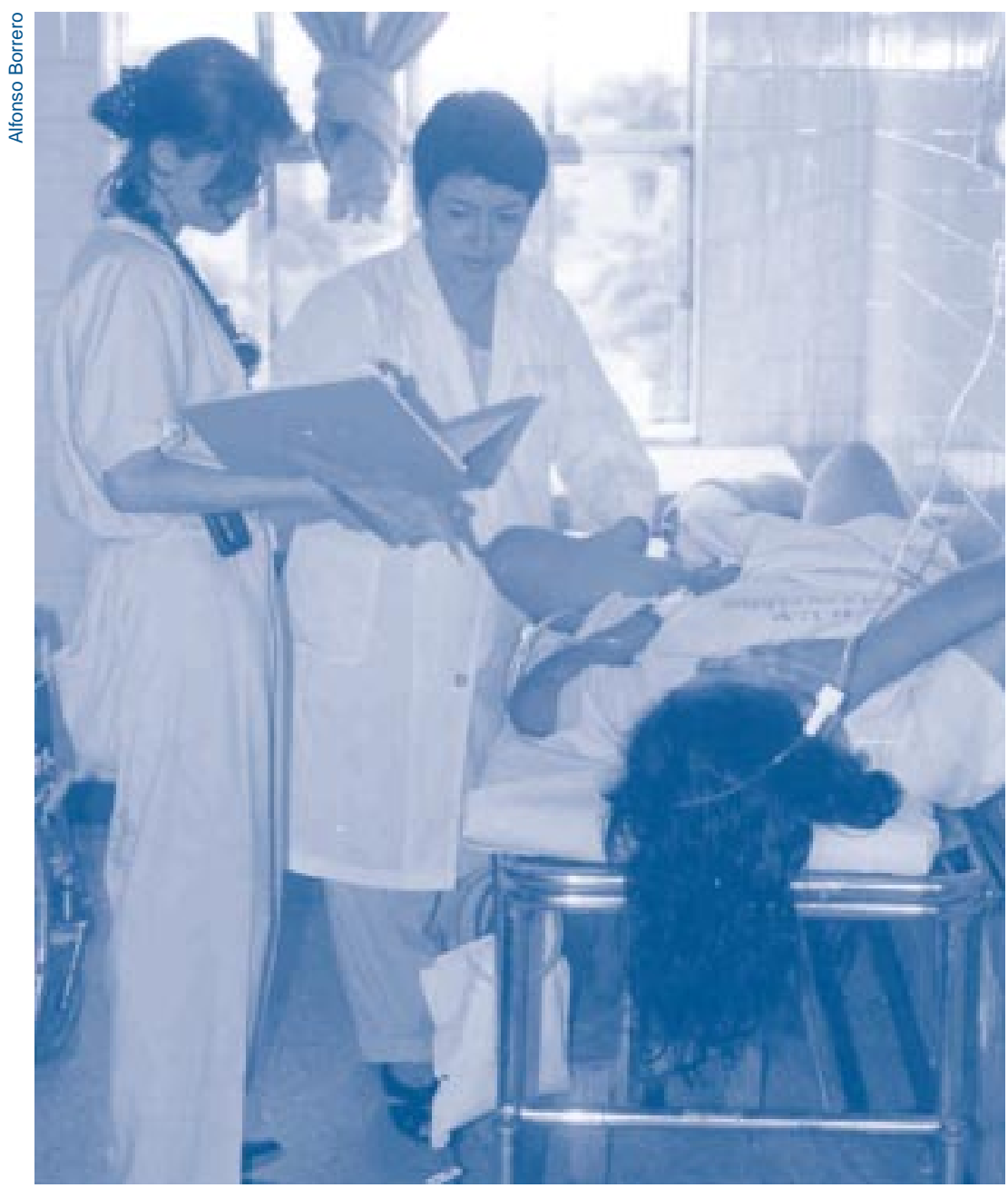

Frontiers in Reproductive Health Program Brief No. 1

Author: Dale Huntington, Sc.D.

Editors: Cynthia P. Green, Ph.D. Stephanie Joyce

Claudine Chen-Young

The assistance of the following reviewers is appreciated:

Janie Benson, Deborah Billings,

Nicole Buono, Sally Girvin,

Lorelei Goodyear, Sarah Harbison, Marjorie Horn, Suellen Miller, Emma Ottolenghi, John Townsend, Ricardo Vernon, Elizabeth Westley, Nancy Williamson, and Beverly Winikoff. Photo on p. 6: courtesy of M/MC Photoshare, www.jhuccp.org/mmc

Suggested citation: Huntington, Dale. 2000. Meeting Women's Health Care Needs after Abortion. Program Briefs. 1. Washington, D.C.: Population Council/FRONTIERS.

Frontiers in Reproductive Health Population Council 4301 Connecticut Ave., N.W.

Suite 280

Washington, D.C. 20008 U.S.A.

Tel.: $\quad 202-237-9400$

Fax: $\quad 202-237-8410$

E-mail: frontiers@pcdc.org www.popcouncil.org

This publication was made possible through support provided by the Office of Population, Bureau for Global Programs, Field Support and Research, U.S. AGENCY FOR INTERNATIONAL DEVELOPMENT (USAID), under the terms of Cooperative Agreement No. HRN-A-00-98-00012. The opinions expressed herein do not necessarily reflect the views of USAID.

Q Population Council 5 Family Health International

Tulane University School of Public Health and Tropical Medicine 
Following are five strategies for improving the quality of PAC:

\section{Upgrade clinical care.}

Programs should strive for the holistic treatment of the patient. Program managers need to develop a systematic plan for providing postabortion care to ensure that patients receive prompt attention, staff time is used efficiently, and quality of care remains high. Developing a systematic care plan need not require large expenditures and can greatly improve patient care. In Kenya, for example, a 1996-97 pilot program tested a service reorganization involving (1) designating small rooms for MVA procedures to free up the main operating rooms; (2) creating private counseling areas; (3) training nurses and physicians on hospital wards in family planning counseling; and (4) providing contraceptive methods. This reorganization reduced patient stays by as much as 60 percent and increased adoption of contraceptive methods by roughly 31 times pre-intervention levels (Solo et al. 1999).

Most PAC patients can be safely managed as outpatients. Shifting to outpatient PAC services (when appropriate) can also make services more efficient and reduce costs (see Strategy 4. Plan comprehensive PAC services).

Constant vigilance is needed to maintain high standards of care. Where staff turnover is high, new staff must receive the appropriate service protocols and training. All staff need regular supervision.
The essential elements of highquality clinical services include:

\section{Use of technology appropriate for the setting and the patient}

Appropriate pain management before, during, and after the clinical procedure

\section{- Infection prevention prac- ticed by all providers}

Adequate supplies of essential medications and surgical equipment

\section{Improved client-provider inter- actions.}

These elements are discussed in more detail below.

\section{Use appropriate technology. In} many settings, the introduction of manual vacuum aspiration, combined with reorganization of services, has led to lower complication rates and shorter patient stays. Numerous studies in the U.S. and developing countries show that MVA is a safer, equally effective, and less resourceintensive treatment for incomplete abortions of up to 12 weeks gestation compared with sharp curettage or dilation and curettage (D\&C), which is the standard treatment in many countries (Greenslade et al. 1993). With properly trained personnel, MVA can be used in primary health care centers and health posts. Studies in Burkina Faso, Egypt, Kenya, Mexico, Peru, and Senegal showed that after receiving training in MVA, providers preferred MVA in most or all uncomplicated cases (PC/Africa OR/TA 1999; PC/INOPAL 1998; Huntington et al. 1995). The introduction of MVA also serves as a catalyst for making improvements to emergency obstetric care. By drawing providers' attention to a "new" surgical device, ancillary services such as pain control, counseling, and linkages with family planning can be reinforced.

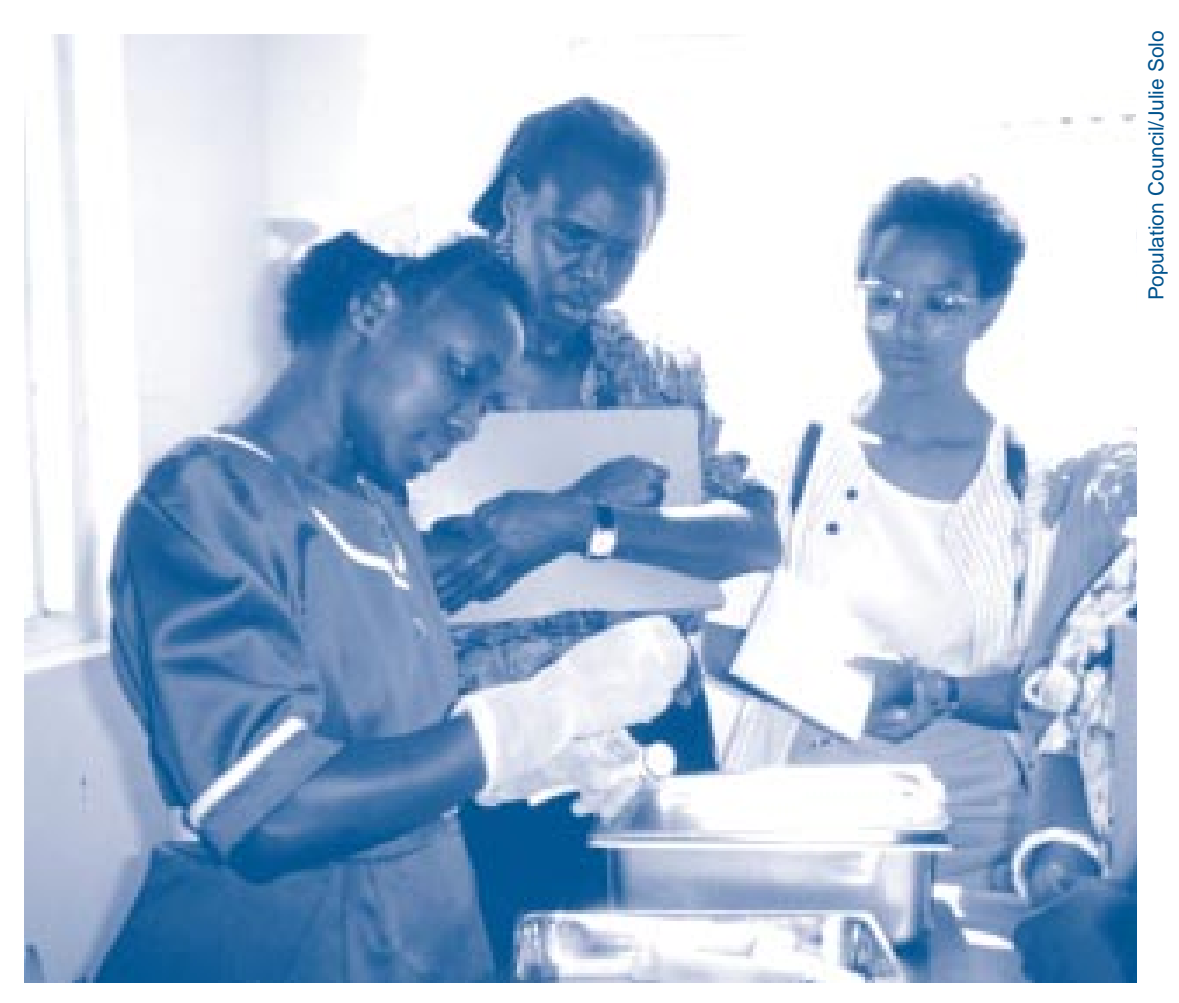




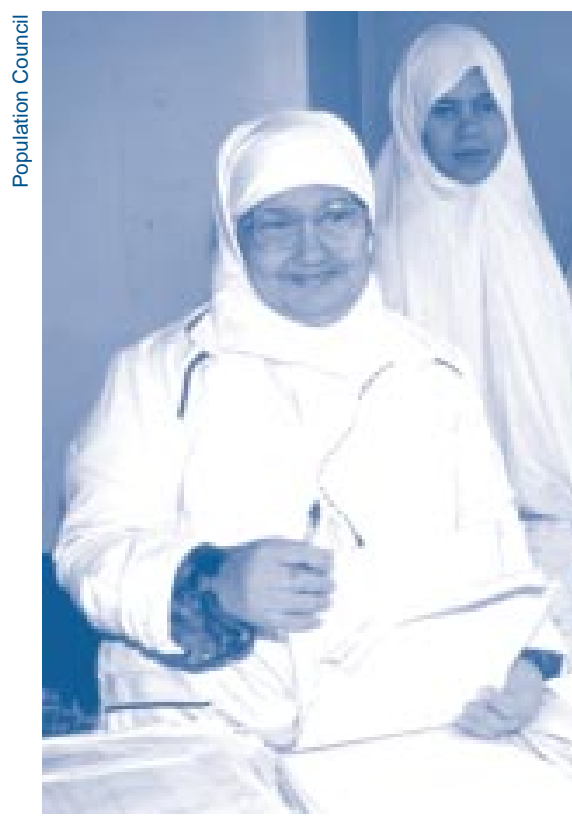

However, program managers seeking to improve postabortion care should weigh the advantages of introducing new techniques such as MVA versus the benefits of improving established practices. In settings where $\mathrm{D} \& \mathrm{C}$ is already institutionalized, providers are often unfamiliar with MVA and thus require additional training. MVA entails recurrent costs for special equipment and supplies. Its use may be curtailed by regulations limiting the importation of MVA equipment.

\section{Pay attention to pain manage-}

ment. Postabortion patients experience both pain and fear before, during, and after uterine evacuation (Huntington, Nawar and Abdel-Hady 1997; Fuentes Valásquez et al. 1998). Although providers typically sedate or anesthetize women during treatment with $\mathrm{D} \& \mathrm{C}$, many of them do not provide adequate pain medication during MVA.

Furthermore, most providers do

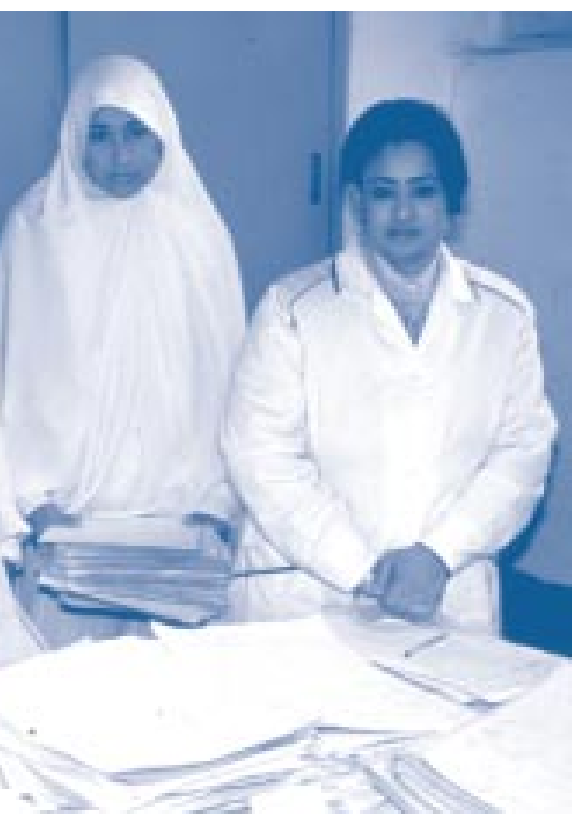

not address the pain or anxiety experienced by women waiting for services or recovering from a procedure. In a Kenya study, only 3 percent of MVA patients and 44 percent of $\mathrm{D} \& \mathrm{C}$ patients received pain medication during the procedure, and providers rarely spoke to the patients during treatment. Three in five of the postabortion patients interviewed described their pain during the procedure as extreme (Solo et al. 1999).
Women's perception of pain may be lessened if they receive verbal support from providers throughout their stay in the health facility. A study in Peru found that training in interpersonal skills helped providers to allay women's fear and anxiety. Hospital patients treated with MVA reported lower pre-procedure pain compared with a baseline group of $\mathrm{D} \& \mathrm{C}$ patients. Patients' perception of pain may also have been affected by the reorganization of clinical services that reduced the wait for service (Benson et al. 1998). Despite these reports of reduced pain, virtually all studies show that many women still experience severe pain during postabortion treatment, underscoring the need to implement pre-, intra-, and post-operative pain management protocols and train providers in interpersonal skills to lessen fear and anxiety.

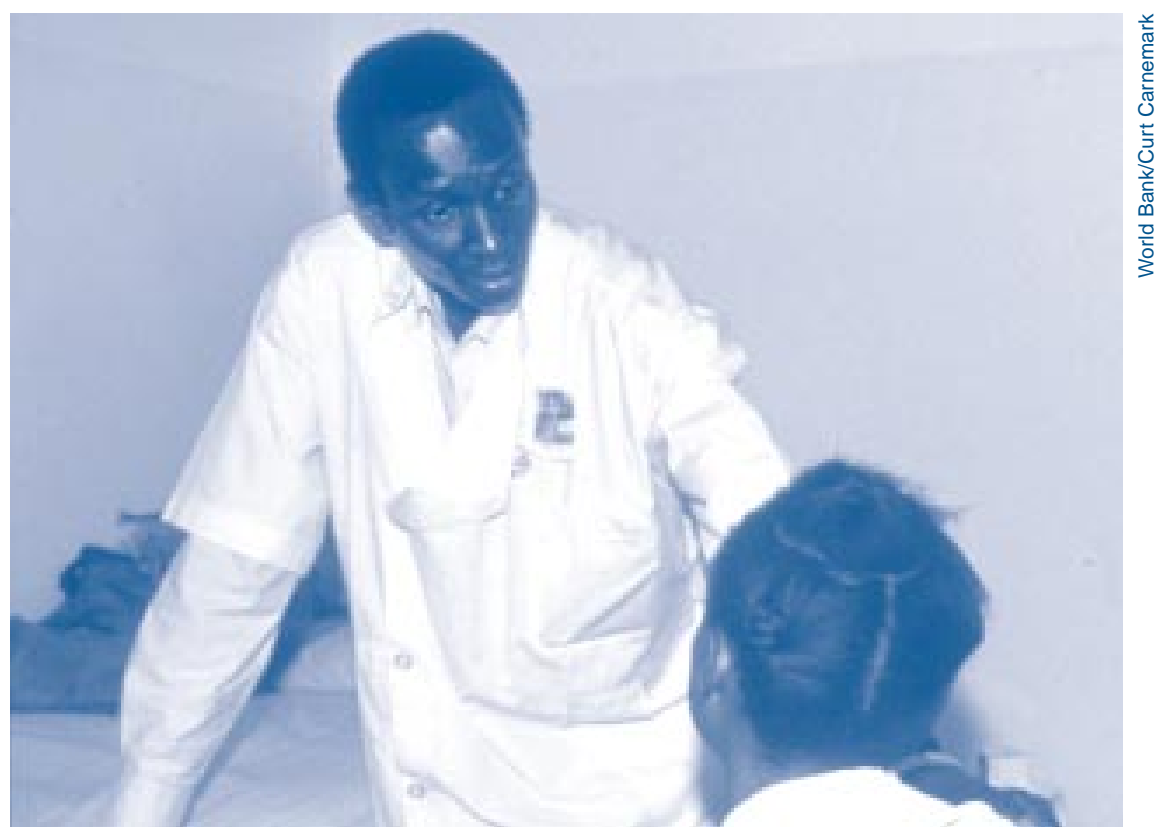




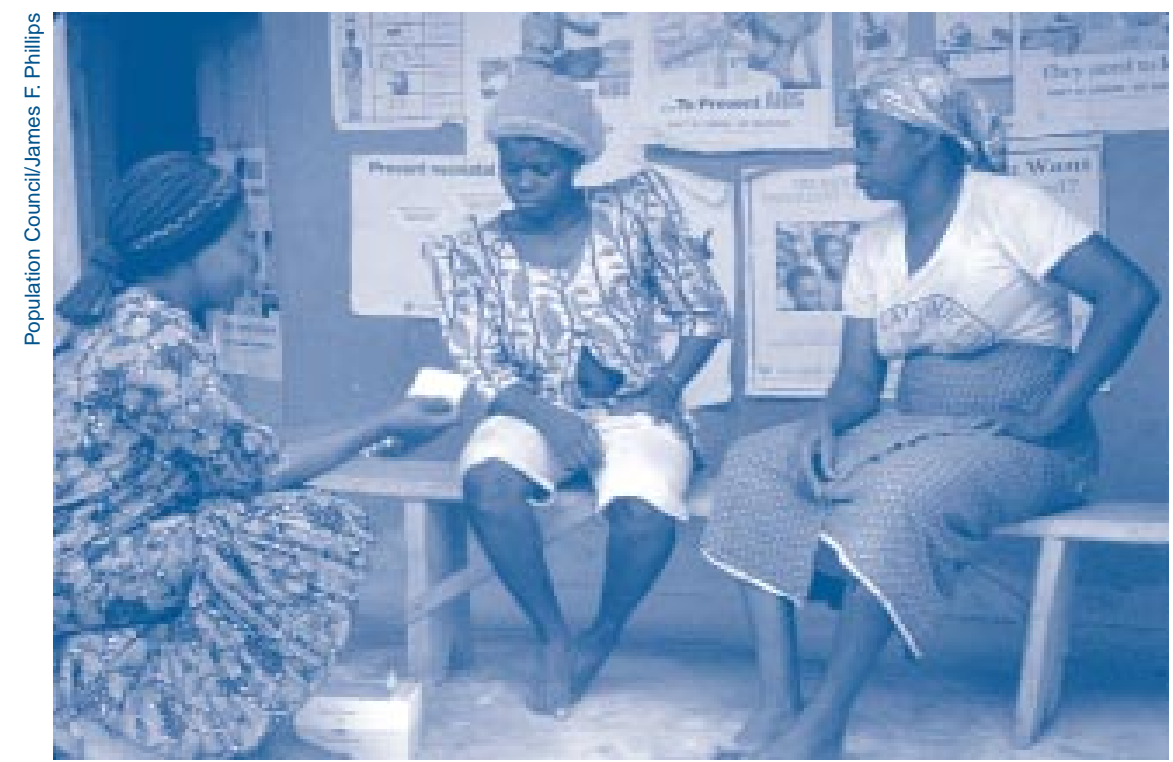

Practice infection prevention.

Key infection prevention practices consist of handwashing, use of gloves, adequate patient preparation, use of the "no-touch" technique, decontamination, highlevel disinfection or sterilization of medical instruments, and safe disposal of biohazardous waste (Margolis, Leonard and Yordy 1993; King et al. 1998b). Infection prevention is simple and inexpensive, yet it remains a problem in many clinical settings. Studies in Bolivia, Honduras, Mexico, and Peru found that infection prevention practices were inadequate in many hospitals, despite the availability of appropriate protocols (King et al. 1998b). Program managers need to ensure that all service points. In the public providers have sufficient training in infection prevention, supervision, and supplies to maintain high standards.

\section{Ensure adequate supplies.}

To maintain high-quality PAC services, medicines and supplies must be continually available. Pre-intervention assessments in several countries found many sites without the necessary equipment, including specula, gloves, sterilizers, and examining tables. Periodic stockouts of medicines and disinfectants are common in many countries. Program managers need to ensure that equipment and supplies are on hand in sector, the necessary supplies need to be on the $\mathrm{MOH}$ procurement list so that health facilities can order them. MVA equipment is usually obtained from a local medical supply distributor; the distributor may need to obtain government approval for the importation and sale of MVA equipment.
Promote improved clientprovider interactions. In many settings, some providers harbor negative attitudes toward women who have had an abortion. These views prevent them from treating PAC patients compassionately and sensitively. Differences in gender, social class, and language can also lead to strained relations between providers and patients. Training in quality of care and interpersonal skills can help to change providers' attitudes and motivate them to improve their interactions with patients. In Mexico, after providers attended a twoday workshop on interpersonal relations and other improvements in the quality of care were introduced, postabortion patients received significantly more information about follow-up care. The proportion of patients who were satisfied with the information they received rose from 18 percent before the intervention to 73 percent after the intervention. Postabortion patients reported significant improvements in many aspects of family planning counseling, including the information provided and the counselor's treatment of them (Langer et al. 1999). 


\section{Provide family planning information and services.}

Offer family planning counseling and services to postabortion

patients. In many cases, women seeking emergency treatment for abortion complications are discharged without adequate information about family planning and may have difficulty accessing family planning services. By making family planning information and services readily available to postabortion patients, providers can help to prevent future unwanted pregnancies.

Offering family planning at the time of emergency treatment is important because women's fertility can return within two weeks after abortion. Thus many postabortion patients have an immediate need for family planning to prevent another unwanted pregnancy. Studies in Bolivia, Burkina Faso, Kenya, Mexico, Peru, and Senegal have demonstrated that after improved PAC services were introduced, the proportion of patients who were counseled about family planning increased (see Figure 1).

Furthermore, most patients who received counseling chose to use contraception (see Figure 2).

\section{Figure 1: Percentage of PAC Patients Who Received Family Planning Counseling}

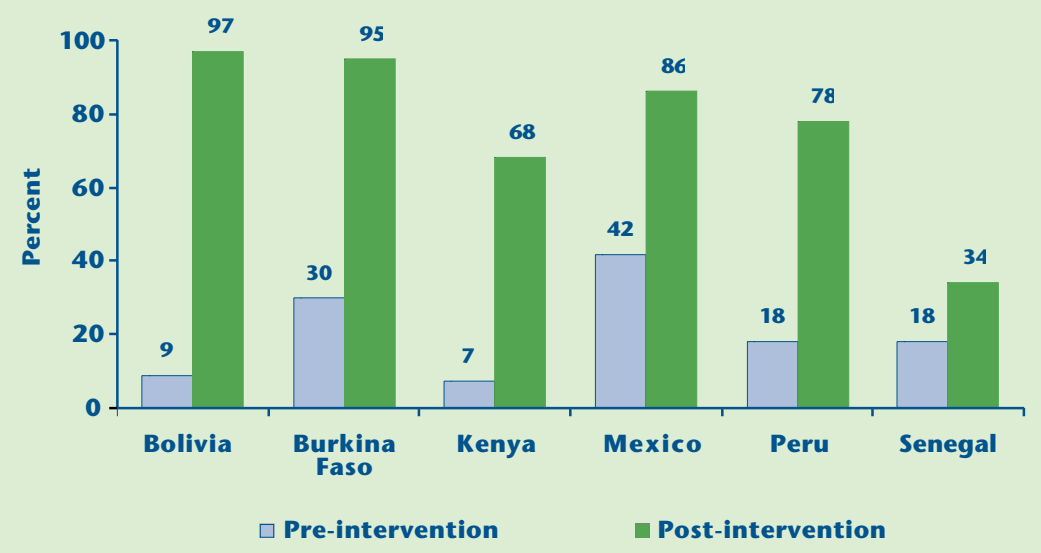

Sources: Bolivia (PC/INOPAL 1998); Burkina Faso (MOH 1998); Kenya (PC/Africa/OR/TA 1999); Mexico (Langer et al. 1999); Peru (Benson et al. 1998); Senegal (CEFOREP 1998).

\section{Figure 2. Percentage of PAC Patients Counseled Who Obtained a Contraceptive Method}

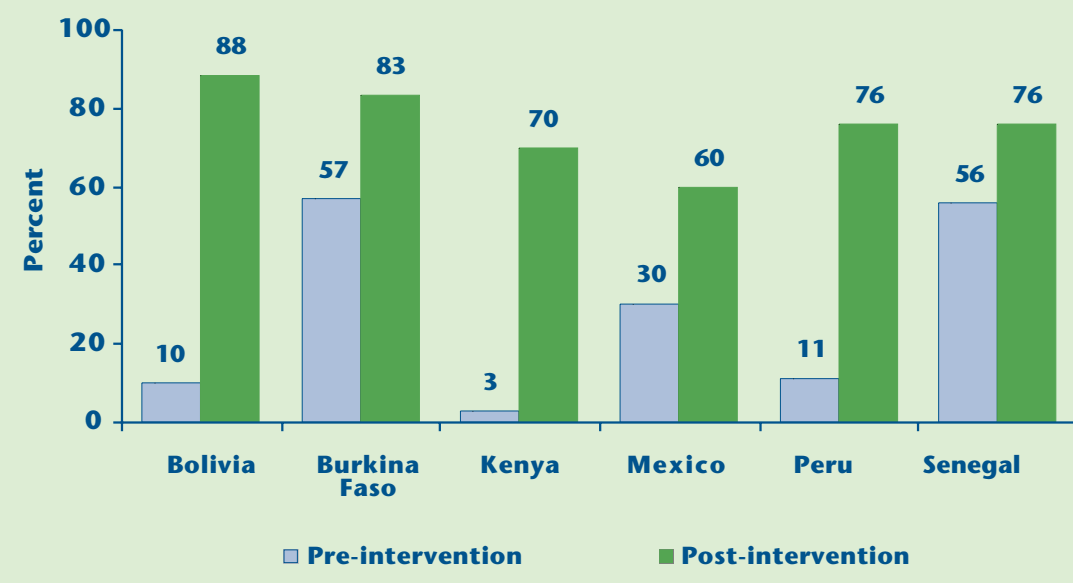

Sources: Bolivia (PC/INOPAL 1998); Burkina Faso (MOH 1998); Kenya (PC/Africa/OR/TA 1999); Mexico (Langer et al. 1999); Peru (Benson et al. 1998); Senegal (CEFOREP 1998). 
Consolidating services to use space and staff time efficiently can make family planning services more accessible. After services were reorganized in six Kenya hospitals, 68 percent of PAC patients received FP counseling, compared with 7 percent before the change. This study found that having ward staff provide family planning information and services was more convenient than having maternal and child health/family planning $(\mathrm{MCH} / \mathrm{FP})$ providers come to the ward or taking patients to the hospital's MCH/FP clinic. When ward staff provided family planning services, 92 percent of the postabortion patients received family planning counseling, compared with 63 percent of those on wards visited by $\mathrm{MCH} / \mathrm{FP}$ staff and 54 percent of those who had to be taken to the hospital's MCH/FP clinic for counseling (Solo et al. 1999).

\section{Expand access to PAC.}

Train community health workers to provide family planning and make referrals. Making comprehensive PAC services available in remote rural areas can save lives and help to protect women's health. Health workers can play an important role by providing basic reproductive health services, including family planning, and by linking women to emergency obstetrical care and other health services.

\section{Make PAC services available at the community level. In many settings, physicians specializing in obstetrics and gynecology pro- vide emergency treatment for abortion complications, thereby restricting PAC services to hospi- tals. However, PAC is a simple}

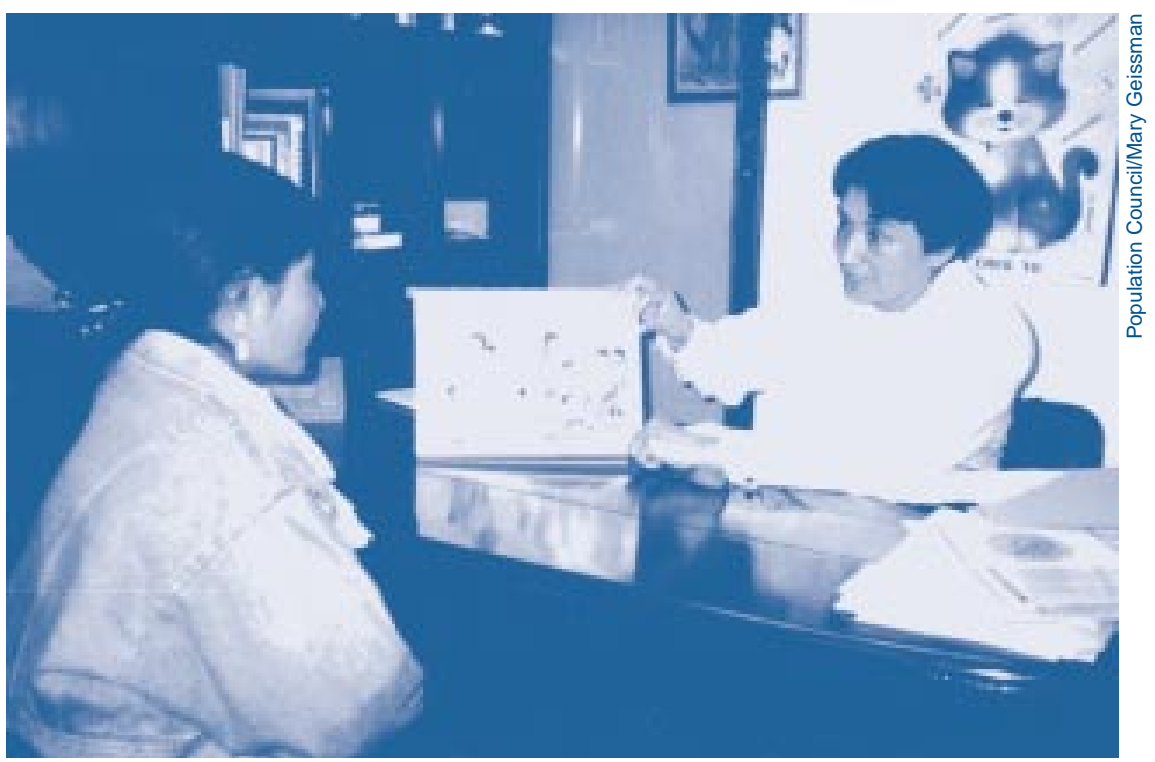

emergency treatment, and if care protocols are followed, it can be provided in community clinics and health centers.

\section{Train mid-level practitioners to perform MVA. Several studies} have shown that emergency treatment using MVA can be safely provided in lower-level facilities by mid-level practitioners such as professional nurses and midwives. A 1996-98 study in Ghana, funded by MotherCare/John Snow Inc., found that professional midwives with special training could perform MVA safely in rural health and maternity centers. Postabortion patients receiving treatment from midwives had shorter waiting times and lower out-of-pocket costs than those treated by physicians in district hospitals. Postabortion patients treated by midwives were more likely to receive family planning counseling and to adopt contraception than other postabortion patients (Billings et al. 1999).
Improve referral systems for reproductive health services. Prompt referral for obstetrical emergencies, including abortion complications, can save lives. In rural Pakistan, a community-based referral program significantly reduced overall maternal mortality by training traditional birth attendants to refer women with obstetric complications to medical facilities (Bashir 1991).

However, many areas lack referral systems, and many women do not know where to obtain emergency care for abortion complications. Two-thirds of the women interviewed for a study in Senegal said that they had visited two or more hospitals before receiving treatment, delaying care for up to five days after the onset of symptoms (CEFOREP 1998). Links among reproductive care centers, emergency clinics, family planning organizations, and community networks can guide women to the most appropriate clinical setting for emergency treatment and other reproductive health services, including treatment of sexually transmitted infections. 


\section{Plan comprehensive PAC services.}

\section{Plan PAC services to improve} quality of care and cut health care costs. Many hospitals consistently provide postabortion care on an unplanned, emergency basis. A study in Egypt showed that one out of every five $\mathrm{Ob} / \mathrm{Gyn}$ admissions was for treatment of complications from an incomplete abortion

(Huntington et al. 1998). Where rates of postabortion complications are high, poorly planned services constitute a considerable drain on health care resources. For example, in Bolivia, health officials estimate that 60 percent of the costs of obstetric/gynecological wards stem from postabortion treatment (Friedman et al. 1999).

Instituting strategically planned postabortion care can improve treatment while consuming fewer resources. A 1998 overview of 21 studies in six countries showed that strategies to improve postabortion care shortened patient stays by an average of 30 percent and reduced treatment costs by 61 percent (King, Benson and Stein 1998a). Studies in Burkina Faso and Senegal also showed that improving PAC services shortened hospital stays by roughly 50 percent, thereby reducing hospital costs per patient by 56 percent and 25 percent, respectively (see Figure 3 ).

After a large hospital in Oaxaca, Mexico reorganized its PAC services and introduced MVA, the average cost of treating a postabortion patient dropped by nearly onethird, from US\$264 to US\$180. The cost of adding family plan-

\section{Figure 3. Cost of Improved PAC Services (US\$)}

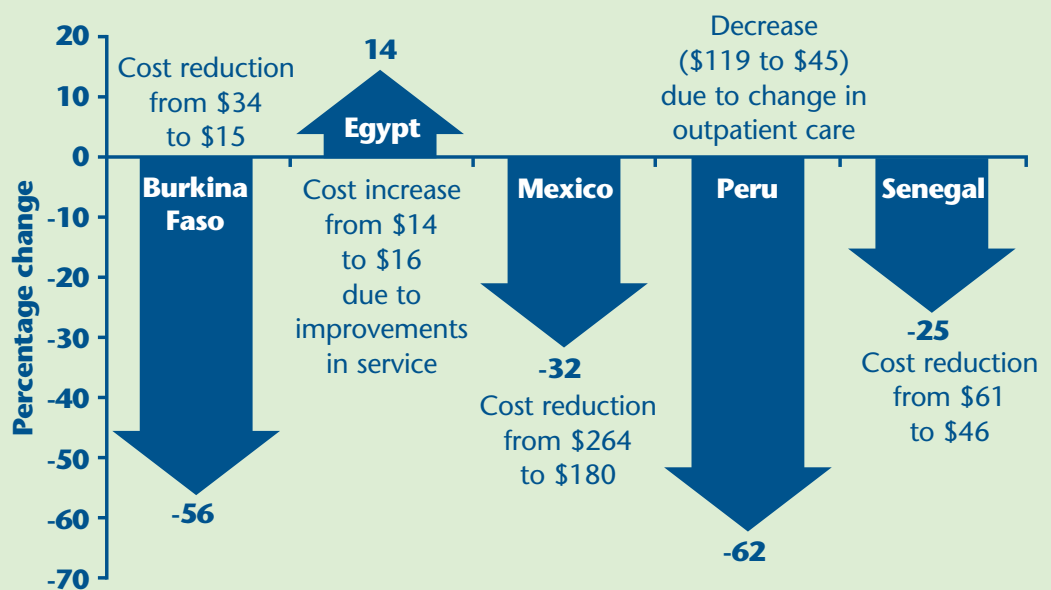

Sources: Burkina Faso (MOH 1998); Egypt (Nawar, Huntington and Fattah 1999); Mexico (Fuentes Valásquez et al. 1998); Peru (Benson et al. 1998); Senegal (CEFOREP 1998).

ning services (including supplies) was about US\$3 per patient (Brambila et al. 1999).

However, improving PAC services does not always reduce costs. In Egypt, the service reorganization at two hospitals entailed raising standards of patient care, such as introducing procedures for pain control, patient counseling, and aseptic conditions. These improvements led to modest increases in costs (Nawar, Huntington, and Fattah 1999).

\section{Offer outpatient PAC services.}

Where it is feasible and appropriate for individual patients, providing postabortion care on an outpatient basis can achieve significant savings. After a hospital in Callao, Peru shifted to outpatient PAC services, patients stayed six hours on average, compared with 33 hours when only inpatient services were available - an 81 percent reduction in average patient stay. The savings in shorter patient stays made up for the increased costs for staffing and supplies - 13 percent and 44 percent, respectively. In light of these savings, the hospital cut patient fees for PAC services by half. The reorganization of services had other benefits, including improved provider-patient relationships and more convenient access to family planning methods (Benson et al. 1998).

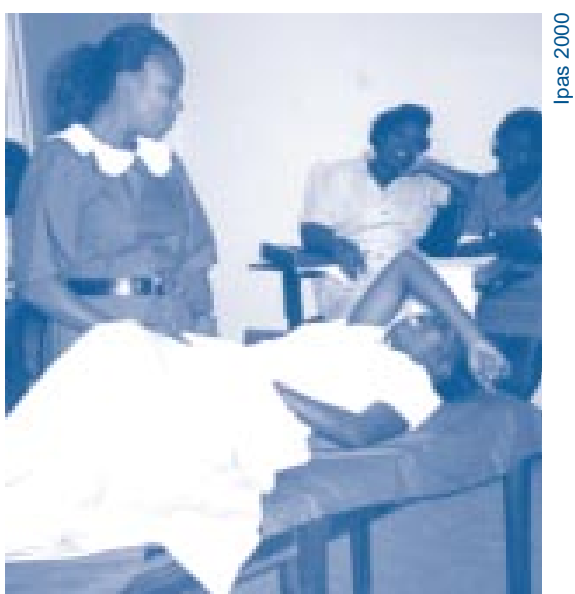




\section{Involve male partners.}

Inform male partners about PAC treatment and follow-up care.

Although men frequently accompany their partners to hospitals for postabortion care, health providers often ignore them and fail to provide even basic information about the woman's condition and postoperative care. Talking with male partners or counseling couples together, when the woman gives prior consent, can help men understand how to help their partner recuperate after the procedure, decide on appropriate family planning methods, and ultimately prevent further unplanned pregnancies and repeat abortions. A 1997 study in Egypt found that couples in which the husband had been counseled by a physician were more likely to be using or planning to use contraception one

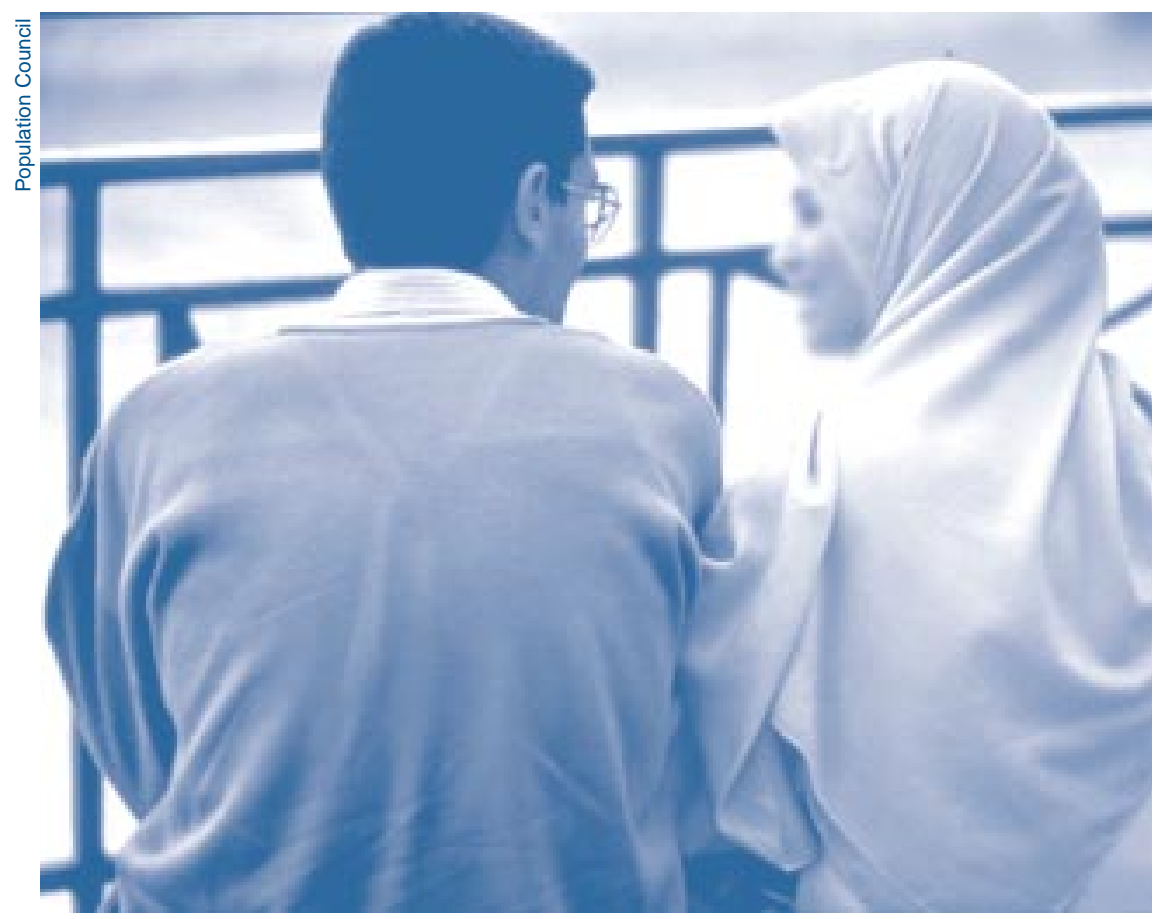

\section{Can improved postabortion care be sustainable?}

(Abdel-Tawab et al. 1999). In Senegal, nearly two-thirds (65\%) of the PAC patients interviewed said that they would like their husband or partner to be present during family planning counseling. A 1995 study in a Turkish hospital found that almost 98 percent of couples who received family planning counseling chose a contraceptive method after abortion, and men who had participated in counseling were more likely to opt for vasectomy, compared with those who had no counseling (Blaney 1997).

Nevertheless, the wishes of women who do not want their partners involved - such as 35 percent of the women in the Senegal study - must also be respected.
Program managers need to ensure that improved PAC services continue over time. A study at a hospital in Callao, Peru identified five critical factors likely to affect continuation of services:

1. The hospital's willingness to commit funds and staff time to make the necessary improvements and pay for contraceptives and other supplies

\section{Improved physical facilities}

3. Incorporation of PAC into routine training for obstetricsgynecology staff

4. Hospital staff support for the improvements

5. Political support from the Ministry of Health, which helped design the intervention and disseminate its findings to other organizations (Benson et al. 1998).

Institutionalizing systematic postabortion care will likely require creation of partnerships with safe motherhood programs and family planning clinics, as well as continued expansion of the range of informed constituencies within medical communities (Huntington 1998). 


\section{What can policymakers do to improve and expand PAC services?}

\section{Policymakers can:}

Support high-quality, women-centered services.

Develop national norms and standards regarding PAC, including pain management, infection prevention, and family planning counseling and services.

Decentralize services to extend access and care to rural women and those living in marginal urban areas and to reduce congestion in busy city hospitals.

Include the cost of PAC instruments and supplies, including MVA kits, and pain medications in the regular budgets of health programs.

Develop local training capability to ensure that health care providers working in reproductive health are routinely trained in PAC as a comprehensive care model, including MVA techniques, pain management, infection prevention, postabortion FP, counseling skills, and referrals to other RH services.

Provide supportive supervision systems to ensure and maintain quality services.

\section{What are the priorities for PAC research?}

Although much knowledge has been generated recently, several unexplored or under-explored areas remain (Billings 1998). Priority topics for future study include:

Decentralizing services. Most operations research studies have taken place in hospitals, typically in urban areas. As services move out of hospitals to health centers and the primary care level, there is increasing need to understand the required inputs to support paramedical personnel who provide postabortion services in outlying areas. In particular, the procedures for maintaining quality services - such as supportive supervision - need to be identified.

\section{Counseling family members and mobilizing social support} systems. Despite increasing awareness that involving men can help to improve women's reproductive health status, few service delivery programs have done so. Further exploration of specific strategies to involve partners or other concerned family members is an important direction for gender-sensitive programs.

\section{Cost savings resulting from improved PAC services. Studies} to date have not addressed issues beyond immediate costs for postabortion care. New research is needed on ways to reallocate the resources freed by improved care and on the significance of savings to hospital managers as well as to the woman and her family.
Ensuring the supply of MVA equipment and essential medications. The MVA technique has been proven successful and costeffective in some settings. More work is needed to ensure the local commercial availability of MVA equipment, which is often constrained by local regulations, and to ensure that health systems have a systematic and uninterrupted supply of MVA instruments and materials for instrument disinfection or sterilization.

Women's perceptions of abortion and unwanted pregnancy.

An understanding of the interrelationships among contraceptive use dynamics (including emergency contraception), unwanted pregnancy, and induced abortion is central to breaking a cycle of repeat abortions. More qualitative research on these issues is needed, particularly studies that suggest programmatic interventions that respond to women's perceptions and needs as well as their social context.

Scaling-up strategies, dissemination of successful models, and advocacy. A great deal of research has been conducted on postabortion care; now it is important to expand dissemination and advocacy efforts to maximize the benefits from the research.

Postabortion follow-up studies. There have been only a few long-term follow-up studies of postabortion patients, in part reflecting the difficulty, sensitivity, and ethical complexity of such studies. More work is needed, particularly, to document the effects of providing contraceptives in terms of reducing repeat abortions. 
- Provider barriers. Studies are needed to determine which provider attitudes and practices constitute barriers to improved PAC services and postabortion family planning.

\section{口 Linking PAC to other RH serv-}

ices. Research is needed to examine the linkages between treatment of abortion complications and related reproductive health care. In regions with high prevalence of HIV and other sexually transmitted infections, these linkages are especially critical to the well-being of women who seek PAC.

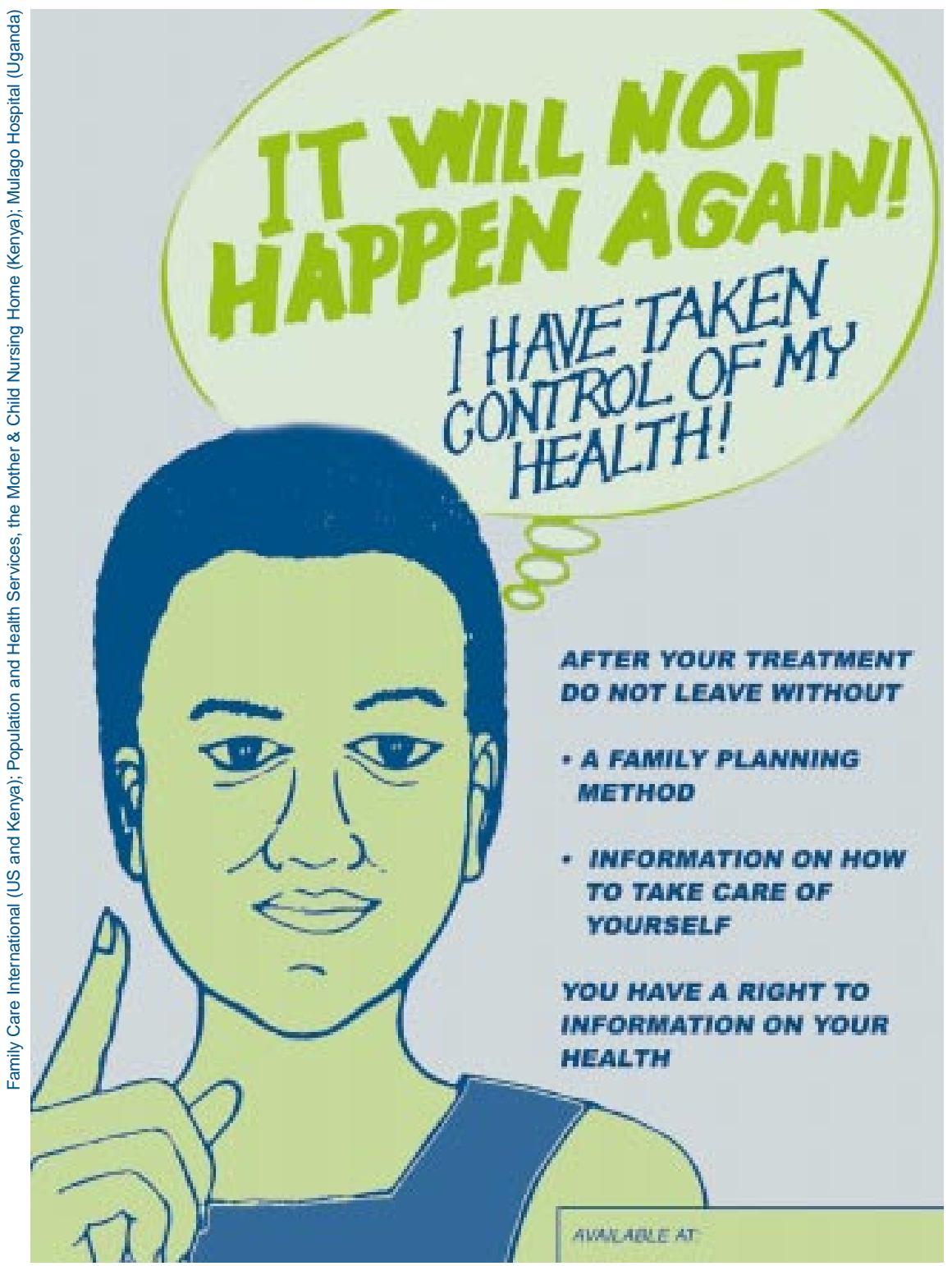

\section{Conclusions}

The basic models for providing high-quality postabortion care have been tested in diverse settings and found to be successful and cost-effective. Once these models have been introduced, service providers and program managers readily appreciate their benefits for improving the quality of care for women with abortion complications. These benefits extend into other areas of reproductive health care and hospital procedures.

Policymakers play a critical role in ensuring that opinion leaders, medical authorities, legal experts, and the general public understand the need for postabortion services as well as the importance of making contraceptive information and services widely accessible in order to prevent unwanted pregnancies. Providing comprehensive treatment and counseling to postabortion patients is the first step in saving women's lives, reducing repeat unsafe abortions, helping women to cope with miscarriages, and improving women's overall health. 


\section{Resources for Program Managers}

Baird, T.L., M.V. Chambers, and C.E. Hord. 1998. "Implementing Postabortion Care." Technical Resources for Postabortion Care. Vol. 1. Carrboro, NC: Ipas. A basic reference for program managers.

Brazier, Ellen, Rahna Rizzuto, and Merrill Wolf. 1998. Prevention and Management of Unsafe Abortion: A Guide for Action. New York: Family Care International. A compendium of resources for program managers.

Ghosh, Anita, Dana Lewison, and Enriquito R. Lu (eds.). 1999. Issues in Establishing Postabortion Care Services in Low-Resource Settings. Baltimore: JHPIEGO Corporation. An overview of strategies to expand PAC.

Hord, Charlotte E., Traci L. Baird, and Debbie L. Billings. 1999. "Advancing the Role of Midlevel Providers in Abortion and Postabortion Care: A Global Review and Key Future Actions." Issues in Abortion Care 6. Chapel Hill, NC: Ipas. Guidelines for supporting mid-level providers.

Huntington, Dale. 1998. Advances and Challenges in Postabortion Care Operations Research. New York: Population Council. A summary of research on PAC programs.
Huntington, Dale and Nancy J. Piet-Pelon (eds.). 1999. Postabortion Care: Lessons from Operations Research. New York: Population Council. A collection of operations research studies on PAC.

Otsea, K. et al. 1999. "Monitoring Postabortion Care." Technical Resources for Postabortion Care. Vol. 3. Chapel Hill, NC: Ipas. A guide for developing monitoring systems.

Salter, Cynthia, Heidi Bart Johnston, and Nicolene Hengen. 1997. "Care for Postabortion Complications: Saving Women's Lives." Population Reports. Series L, No. 10. Baltimore: Johns Hopkins School of Public Health, Population Information Program, September. A comprehensive overview of PAC and a training resource.

World Health Organization. 1997. Postabortion Family Planning: A Practical Guide for Programme Managers. Geneva: WHO Division of Reproductive Health (Technical Support). A detailed guide to strengthening PAC programs. 


\section{References}

Abdel-Tawab, Nahla et al. 1999. "Effects of Husband Involvement on Postabortion Patients'

Recovery and Use of Contraception in Egypt," in Postabortion Care: Lessons from Operations Research, ed. D. Huntington and N.J. Piet-Pelon. New York: Population Council, pp. 16-37.

\section{Bashir, A.1991. "Maternal} Mortality in Pakistan: A Success Story of the Faisalabad District," IPPF Medical Bulletin 25(2):1-3, April.

Benson, Janie et al. 1998. Improving Quality and Lowering Costs in an Integrated Postabortion Care Model in Peru: Final Report. Lima, Peru: Ipas and Population Council.

Billings, Deborah L. 1998. "Integration of Postabortion Care Services with Other Reproductive Health Services." Position Paper for Frontiers in Reproductive Health.

Washington, DC: Population Council.

Billings, Deborah et al. 1999. "Midwives and Comprehensive Postabortion Care in Ghana," in Postabortion Care: Lessons from Operations Research, ed. D. Huntington and N.J. Piet-Pelon. New York: Population Council, pp.141-156.

Blaney, Carol Lyn. 1997. "Involving Men after Pregnancy," FHI Network. Summer, p. 24.
Brambila, Carlos et al. 1999.

"Estimating Costs of Postabortion Services at Dr. Aurelio Valdivieso General Hospital, Oaxaca, Mexico," in Postabortion Care: Lessons from Operations Research, ed. D. Huntington and N.J. Piet-Pelon. New York: Population Council, pp. 108-124.

Centre de Formation et de Recherche en Santé de la Reproduction (CEFOREP) and Clinique Gynecologique et Obstetricale Chu. A. le Dantec. 1998. Introduction des Soins Obstetricaux d'Urgence et de la Planification Familiale pour les Patientes Presentant des Complications Liées a un Avortement Incomplet. Dakar, Senegal: Population Council.

Friedman, Alison et al. 1999. An Assessment of Postabortion Care (PAC) Services in the Bolivian Public Health System. Mexico City: Population Council, p. 2.

Fuentes Valásquez, Jaime et al. 1998. A Comparison of Three Models of Postabortion Care in Mexico: Final Report. Mexico City: IMSS, Ipas, and Population Council.

Greenslade, Forrest C. et al. 1993. Manual Vacuum Aspiration: A Summary of Clinical and Programmatic Experience Worldwide. Carrboro, NC: Ipas.
Huntington, Dale et al. 1995. "Improving the Medical Care and Counseling of Postabortion Patients in Egypt," Studies in Family Planning 26(6):350-362.

Huntington, Dale, Laila Nawar, and Dalia Abdel-Hady. 1997. "Women's Perceptions of Abortion in Egypt," Reproductive Health Matters, No. 9, May.

Huntington, Dale. 1998. Advances and Challenges in Postabortion Care: Summary Report of a Global Meeting. New York: Population Council, pp. 22-27.

Huntington, Dale et al. 1998. "The Postabortion Caseload in Egyptian Hospitals: A Descriptive Study," International Family Planning Perspectives 24(1):25-31.

Huntington, Dale and Nancy J. Piet-Pelon (eds.). 1999.

Postabortion Care: Lessons from Operations Research. New York: Population Council.

King, Tim, Janie Benson, and Karen Stein. 1998a. "Comparing the Cost of Postabortion Care in Africa and Latin America: The DATAPAC Project," paper presented at the Global Meeting on Postabortion Care Operations Research, New York, Population Council, January. 
King, Timothy et al. 1998b. "Postabortion Care in Latin America: A Summary of Operations Research," in Reproductive Health Operations Research. Mexico City: Population Council/INOPAL III, pp. 59-79.

Langer, Ana et al. 1999. "Improving Postabortion Care with Limited Resources in a Public Hospital in Oaxaca, Mexico," in Postabortion Care: Lessons from Operations Research, ed. D. Huntington and N.J. PietPelon. New York: Population Council, pp. 81-107.

Margolis, Alan, Ann H. Leonard, and Laura Yordy, 1993. "Pain Control for the Treatment of Incomplete Abortion with MVA," Advances in Abortion Care 3(1):1-8.

Ministry of Health, Burkina Faso. 1998. Introduction of Emergency Medical Treatment and Family Planning Services for Women with Complications from Abortion in Burkina Faso. Ougadougou, Burkina Faso: Population Council.

Nawar, Laila, Dale Huntington, and Mohammed Naguib Abdel Fattah. 1999. "Cost Analysis of Postabortion Services in Egypt," in Postabortion Care: Lessons from Operations Research, ed. D. Huntington and N.J. Piet-Pelon. New York: Population Council, pp. 125-140.
Population Council/INOPAL III. 1998. Reproductive Health Operations Research: 1995-1998. Mexico City: Population Council/INOPAL III.

Population Council/Africa OR/TA. 1999. Strengthening Reproductive Health Services in Africa through Operations Research: Final Report of the Africa Operations Research and Technical Assistance Project II. Nairobi, Kenya: Population Council, p. 63.

Salter, Cynthia, Heidi Bart Johnston, and Nicolene Hengen. 1997. "Care for Abortion Complications: Saving Women's Lives," Population Reports, Series L, No. 10, September.

Solo, Julie et al. 1999. "Creating Linkages between Incomplete Abortion Treatment and Family Planning Services in Kenya." Studies in Family Planning 30(1):17-27.

United Nations (UN). 1994.

Report of the International

Conference on Population and

Development (Cairo, 5-13

September 1994). New York: UN. 
Frontiers in Reproductive Health

Population Council

4301 Connecticut Ave., N.W.

Suite 280

Washington, D.C. 20008

U.S.A.

Tel.: $\quad 202-237-9400$

Fax: $\quad 202-237-8410$

E-mail: frontiers@pcdc.org www.popcouncil.org

This publication was made possible through support provided by the Office of Population, Bureau for Global Programs, Field Support and Research, U.S. AGENCY FOR INTERNATIONAL DEVELOPMENT (USAID), under the terms of Cooperative Agreement No. HRN-A-00-98-00012. The opinions expressed herein do not necessarily reflect the views of USAID.

\section{(1) Population Council}

